\title{
A Littlewood-Paley-Stein estimate on graphs and groups
}

by

\author{
Nick Dungey (Sydney)
}

\begin{abstract}
We establish the boundedness in $L^{q}$ spaces, $1<q \leq 2$, of a "vertical" Littlewood-Paley-Stein operator associated with a reversible random walk on a graph. This result extends to certain non-reversible random walks, including centered random walks on any finitely generated discrete group.
\end{abstract}

1. Introduction and statement of results. The main result of this paper is a Littlewood-Paley-Stein estimate in $L^{q}, 1<q \leq 2$, for a random walk on a graph. This estimate is the analogue for graphs of a LittlewoodPaley-Stein estimate on manifolds which we now recall. Consider a complete Riemannian manifold $M$ with Riemannian measure $d x$, and the corresponding (positive) Laplace operator $\Delta$, heat semigroup $\left(e^{-t \Delta}\right)_{t \geq 0}$ and gradient operator $\nabla$. The "vertical" Littlewood-Paley operator $\mathcal{H}_{\Delta}$ is defined for functions $f \in C_{0}^{\infty}(M)$ by

$$
\left(\mathcal{H}_{\Delta} f\right)(x):=\left(\int_{0}^{\infty} d t\left|\left(\nabla e^{-t \Delta} f\right)(x)\right|^{2}\right)^{1 / 2}
$$

for $x \in M$. Then $\mathcal{H}_{\Delta}$ extends to a bounded (sublinear) operator in $L^{q}(M ; d x)$ for $q \in(1,2]$, that is, one has the Littlewood-Paley-Stein estimate

$$
\left\|\mathcal{H}_{\Delta} f\right\|_{q} \leq c_{q}\|f\|_{q}
$$

for all $f \in C_{0}^{\infty}(M)$, with $c_{q}>0$ a constant depending on $q \in(1,2]$. This result is proved in [6, Theorem 1.2], by an argument essentially due to Stein [15]. Inequality (1) does not necessarily hold when $q \in(2, \infty)$. On the other hand, the "horizontal" Littlewood-Paley operator, whose definition involves a time derivative instead of the gradient, is bounded in $L^{q}$ for all $q \in(1, \infty)$ in the context of any symmetric submarkovian semigroup (see [15, Chapter IV]). See $[6,15,11,12]$, and their references, for expositions of further topics in Littlewood-Paley theory.

2000 Mathematics Subject Classification: 42B25, 60G50, 60B15.

Key words and phrases: Littlewood-Paley operator, reversible random walk, graph, centered random walk, gradient. 
Stein's argument for (1) relies on the identity

$$
\Delta\left(f^{q}\right)=q f^{q-1}(\Delta f)-q(q-1) f^{q-2}|\nabla f|^{2}
$$

valid for $q>1$ and non-negative smooth functions $f$ on $M$. Identity (2) depends on the fact that $\Delta$ is locally a second-order differential operator.

The basic problem on graphs is that identities such as (2) are not valid for the discrete Laplace operator on a graph. Thus it is not obvious how to extend Stein's argument to the case of graphs.

To overcome this difficulty, in the present paper we introduce a "pseudogradient" $\Gamma_{q}$, for $1<q \leq 2$, in such a way that Stein's argument yields a Littlewood-Paley type estimate involving $\Gamma_{q}$ in $L^{q}$. Under a weak local uniformity assumption on the graph, we are then able to compare $\Gamma_{q}$ with the gradient to deduce a Littlewood-Paley estimate involving the gradient. Our estimate appears to be new and interesting on graphs, and might be of future use in related problems such as the boundedness of the Riesz transforms on graphs.

Although most of this paper deals with reversible random walks, in Section 5 we observe an extension to certain non-reversible random walks. In particular, we obtain there a Littlewood-Paley-Stein estimate in $L^{q}$, $q \in(1,2]$, for non-reversible, centered random walks on any finitely generated discrete group. It is remarkable that no assumptions on the group structure are needed for this estimate.

Let us describe our setting of a reversible random walk (for further background, see for example [17]). Let $V$ be a countable set and $p: V \times V \rightarrow[0,1]$ a transition probability over $V$, that is, $p(x, y) \geq 0$ and $\sum_{y \in V} p(x, y)=1$ for all $x \in V$. Define $p_{k}$, for $k \in \mathbb{N}=\{1,2,3, \ldots\}$, inductively by $p_{1}:=p$ and

$$
p_{k+1}(x, y):=\sum_{z \in V} p_{k}(x, z) p(z, y), \quad x, y \in V .
$$

We assume that $p$ is irreducible, that is, for any $x, y \in V$ there exists a $k=k(x, y) \in \mathbb{N}$ with $p_{k}(x, y)>0$.

Next, suppose that $m: V \rightarrow(0, \infty)$ is a strictly positive function (measure) which is reversible with respect to $p$, meaning that

$$
m(x) p(x, y)=m(y) p(y, x)
$$

for all $x, y \in V$. For subsets $A \subseteq V$ set $m(A):=\sum_{x \in A} m(x)$. Consider the spaces $L^{q}:=L^{q}(V ; m), q \in[1, \infty]$, with respect to the measure $m$. We use the notation $\|\cdot\|_{q_{1} \rightarrow q_{2}}$ for the norm of a bounded linear operator from $L^{q_{1}}$ to $L^{q_{2}}$. Define the Markov operator $P$ associated with $p$ by

$$
(P f)(x)=\sum_{y} p(x, y) f(y)
$$

for $x \in V$ and suitable functions $f: V \rightarrow \mathbb{C}$ (here and below, summations are 
taken over all elements $y \in V$ unless otherwise indicated). It is a consequence of the reversibility assumption that $P$ is a self-adjoint contraction operator in $L^{2}$, and is a contraction in $L^{q}$ for all $q \in[1, \infty]$. The "discrete Laplacian" $H$, defined by

$$
H:=I-P,
$$

generates a symmetric submarkovian semigroup $\left(e^{-t H}\right)_{t \geq 0}$ in the $L^{q}$ spaces. Note that

$$
e^{-t H}=e^{-t} e^{t P}=e^{-t} \sum_{k \geq 0}(k !)^{-1} t^{k} P^{k}
$$

for $t>0$. For suitable real-valued functions $f$ on $V$, define the gradient $|\nabla f|$ by

$$
|\nabla f|(x)=\left(\sum_{y} p(x, y)(f(x)-f(y))^{2}\right)^{1 / 2}
$$

and the "vertical" Littlewood-Paley function $\mathcal{H} f$ by

$$
(\mathcal{H} f)(x)=\left(\int_{0}^{\infty} d t\left|\nabla e^{-t H} f\right|^{2}(x)\right)^{1 / 2}
$$

for $x \in V$. We say that $\mathcal{H}$ is bounded in $L^{q}$ if there exists $c>0$ with

$$
\|\mathcal{H} f\|_{q} \leq c\|f\|_{q}
$$

for all $f \in L^{1} \cap L^{\infty}$.

To state our main result about $\mathcal{H}$ we need to consider the graph structure on $V$ associated with $p$, as follows. For $x, y \in V$, if $p(x, y)>0$ then we say that $x, y$ are connected by an edge, and write $x \sim y$. By reversibility, $x \sim y$ if and only if $y \sim x$. A path of length $k(k \geq 0)$ joining $x$ to $y(x, y \in V)$ is a sequence $x_{0}, x_{1}, \ldots, x_{k}$ of elements of $V$ with $x_{0}=x, x_{k}=y$ and $x_{j} \sim x_{j+1}$ for $j \in\{0,1, \ldots, k-1\}$. Irreducibility implies that any two points of $V$ can be joined by a path; and the distance $d(x, y)$ is defined to be the minimal length of a path from $x$ to $y$. For $x \in V$ and $r \geq 0$, define the closed ball $B(x, r):=\{y \in V: d(x, y) \leq r\}$.

We say that the triple $(V, p, m)$ satisfies property (LD) if for some constant $c_{0}>1$,

$$
m(B(x, 1)) \leq c_{0} m(x)
$$

for all $x \in V$. ("LD" stands for "local doubling", since $m(x)=m(B(x, 1 / 2)$ ) so that (6) is a type of local volume doubling property for graphs.) It is an easy remark made in $[7$, p. 571$]$ that property (LD) is equivalent to the conjunction of the following two properties:

(i) there exists $c_{1} \geq 1$ such that $m(y) \leq c_{1} m(x)$ for all $x, y \in V$ satisfying $x \sim y$; 
(ii) the graph is uniformly locally finite, in other words $\sup _{x \in V} N_{x}<\infty$, where $N_{x}:=\#\{y \in V: x \sim y\}$ is the number of neighbors of $x$.

We can now state our main theorem.

Theorem 1.1. Let $(V, p, m)$ satisfy property $(\mathrm{LD})$. Then $\mathcal{H}$ is bounded in $L^{q}$ for $q \in(1,2]$. Moreover, for each $q \in(1,2]$ there exists a $c_{q}>0$ such that

$$
\left\|\left|\nabla e^{-t H} f\right|\right\|_{q} \leq c_{q} t^{-1 / 2}\|f\|_{q}
$$

for all $f \in L^{q}$ and $t>0$.

For $q \in(2, \infty), \mathcal{H}$ is not necessarily bounded in $L^{q}$ and (7) might not hold, depending on $(V, p, m)$. A basic counterexample is a standard random walk on a graph formed of two copies of $\mathbb{Z}^{D}$ joined by a single edge; although we omit the details, it is possible to show that (7) fails when $D \geq 3$ and $q>D$. Analogous negative results, for a manifold formed of two copies of $\mathbb{R}^{D}$, were given in [4].

From Theorem 1.1, in fact merely from (7), we shall obtain the following. Let $\sigma_{L^{q}}(A)$ denote the spectrum of an operator $A$ acting in $L^{q}$.

Corollary 1.2. Let $(V, p, m)$ satisfy (LD), and let $q \in(1,2]$. There exists $c_{q}>0$ such that

$$
\||\nabla f|\|_{q} \leq c_{q}\|H f\|_{q}^{1 / 2}\|f\|_{q}^{1 / 2}
$$

for all $f \in L^{q}$. If , in addition, $-1 \notin \sigma_{L^{2}}(P)$, then there exists $c_{q}^{\prime}>0$ such that

$$
\left\|\left|\nabla P^{k} f\right|\right\|_{q} \leq c_{q}^{\prime} k^{-1 / 2}\|f\|_{q}
$$

for all $f \in L^{q}$ and $k \in \mathbb{N}$.

The analogue of inequality (8) on Riemannian manifolds was established in [5, Theorem 4.1].

Inequality (9) may fail even for $q=2$, if $-1 \in \sigma_{L^{2}}(P)$. The simplest example where it fails is the standard random walk on $V=\mathbb{Z}$ given by $p(x, x \pm 1)=1 / 2$ and $m(x)=1$ for $x \in \mathbb{Z}$. On the other hand, a simple sufficient condition for $-1 \notin \sigma_{L^{2}}(P)$ is that $\inf _{x \in V} p(x, x)>0$ (see, for example, [10, Lemma 1.3]).

Essential to our proof of Theorem 1.1 is the following notion of "pseudogradient". For $q \in(1,2]$ and suitable non-negative functions $f \geq 0$, set

$$
\Gamma_{q}(f):=q f(H f)-f^{2-q} H\left(f^{q}\right)=(q-1) f^{2}-q f(P f)+f^{2-q} P\left(f^{q}\right) .
$$

The Markovian nature of $P$ implies that $\Gamma_{q}(f)$ is a non-negative function and is dominated pointwise by a constant multiple of $|\nabla f|^{2}$ : see inequalities (11) below. In general, however, $\Gamma_{q}(f)$ does not dominate $|\nabla f|^{2}$. It is clear 
that $\Gamma_{q}$ is "homogeneous of degree two" in the sense that $\Gamma_{q}(\lambda f)=\lambda^{2} \Gamma_{q}(f)$ when $\lambda \in[0, \infty)$.

The following result is proved essentially by Stein's argument, and does not assume property (LD).

Theorem 1.3. Let $q \in(1,2]$. Define $\mathcal{H}_{q} f$ by

$$
\left(\mathcal{H}_{q} f\right)(x)=\left(\int_{0}^{\infty} d t \Gamma_{q}\left(e^{-t H} f\right)(x)\right)^{1 / 2}
$$

for $x \in V$ and $0 \leq f \in L^{1} \cap L^{\infty}$. Then there exists $c_{q}>0$ such that

$$
\left\|\mathcal{H}_{q} f\right\|_{q} \leq c_{q}\|f\|_{q} \quad \text { and } \quad\left\|\Gamma_{q}^{1 / 2}\left(e^{-t H} f\right)\right\|_{q} \leq c_{q} t^{-1 / 2}\|f\|_{q}
$$

for all $t>0$ and $0 \leq f \in L^{1} \cap L^{\infty}$.

We will deduce Theorem 1.1 from Theorem 1.3 together with a result showing that, under property (LD), $|\nabla f|^{2}$ is dominated by a local average of $\Gamma_{q}(f)$. This domination relies on an interesting explicit expression for $\Gamma_{q}(f)$ found in Lemma 3.2 below.

Let us record some basic remarks on pseudo-gradients and their relation to the gradient $|\nabla f|$.

(a) The definition of $\Gamma_{q}(f)$ is motivated by identity (2) on manifolds. Indeed, if one replaces $H$ by the Riemannian Laplace operator $\Delta$ in (10), then, by (2), the resulting expression is just a constant multiple of $|\nabla f|^{2}$ on a manifold.

To see that $\Gamma_{q}(f)$ and $|\nabla f|^{2}$ are not the same on graphs when $q<2$, consider for example a function $f: V \rightarrow[0, \infty)$ satisfying $f(x)=0$ and $f(y)>0$ for some points $x, y \in V$ with $x \sim y$. Then $|\nabla f|^{2}(x)>0$ but $\Gamma_{q}(f)(x)=0$ for $q \in(1,2)$. Note that, in contrast, a non-negative smooth function $f$ on a Riemannian manifold $M$ must have vanishing gradient at any point where $f(x)=0$.

(b) For suitable non-negative functions $f$ (for example, if $0 \leq f \in L^{\infty}$ ) the calculation

$$
\begin{aligned}
\left(f^{2}-2 f(P f)+P\left(f^{2}\right)\right)(x) & =\sum_{y} p(x, y)\left(f(x)^{2}-2 f(x) f(y)+f(y)^{2}\right) \\
& =\sum_{y} p(x, y)(f(x)-f(y))^{2}
\end{aligned}
$$

shows that $\Gamma_{2}(f)=|\nabla f|^{2}$.

(c) Let us show that

$$
0 \leq \Gamma_{q}(f) \leq(q / 2)|\nabla f|^{2}
$$

for $q \in(1,2)$ and suitable non-negative functions $f$. (An improved version of this inequality will follow from Lemma 3.2 below.) Since $p$ is a transition 
probability,

$$
\sum_{y} p(x, y) f(y) \leq\left(\sum_{y} p(x, y) f(y)^{q}\right)^{1 / q}
$$

for any $x \in V$, which means that $P f \leq\left(P\left(f^{q}\right)\right)^{1 / q}$ pointwise. By the elementary inequality $s t \leq s^{q^{\prime}} / q^{\prime}+t^{q} / q$ valid for all $s, t \geq 0$, where $q^{-1}+\left(q^{\prime}\right)^{-1}=1$, we see that

$$
f^{q-1}(P f) \leq f^{q-1}\left(P\left(f^{q}\right)\right)^{1 / q} \leq \frac{1}{q^{\prime}} f^{q^{\prime}(q-1)}+\frac{1}{q} P\left(f^{q}\right)=\frac{q-1}{q} f^{q}+\frac{1}{q} P\left(f^{q}\right) .
$$

Multiplying this inequality by $q f^{2-q}$ shows that the last expression in (10) is non-negative, proving that $\Gamma_{q}(f) \geq 0$.

To get the other inequality in (11), note that $P\left(f^{q}\right) \leq\left(P\left(f^{2}\right)\right)^{q / 2}$ and apply an elementary inequality to get

$$
f^{2-q} P\left(f^{q}\right) \leq f^{2-q}\left(P\left(f^{2}\right)\right)^{q / 2} \leq \frac{2-q}{2} f^{2}+\frac{q}{2} P\left(f^{2}\right) ;
$$

inserting this in the definition of $\Gamma_{q}(f)$ yields

$$
\Gamma_{q}(f) \leq(q / 2) f^{2}-q f(P f)+(q / 2) P\left(f^{2}\right)=(q / 2)|\nabla f|^{2}
$$

as desired.

2. Proof of Theorem 1.3. The proof is based on Stein's argument in [15, Chapter II]; see also the proof of [6, Theorem 1.2].

For $f \in L^{1}+L^{\infty}$, define the semigroup maximal function $f^{*}$ by $f^{*}(x):=$ $\sup _{t>0}\left|\left(e^{-t H} f\right)(x)\right|$ for $x \in V$. Since $\left(e^{-t H}\right)_{t \geq 0}$ is a symmetric submarkovian semigroup, the following lemma is contained in [15, Section III.3].

Lemma 2.1. For each $q \in(1, \infty]$, there exists $c_{q}>0$ such that

$$
\left\|f^{*}\right\|_{q} \leq c_{q}\|f\|_{q}
$$

for all $f \in L^{q}$.

To prove Theorem 1.3, let $q \in(1,2]$ and consider a function $0 \leq f \in$ $L^{1} \cap L^{\infty}$. Set $u_{t}:=e^{-t H} f$ for all $t>0$. We may assume that $f \neq 0$; it then follows by irreducibility, recalling (5), that $u_{t}(x)>0$ for all $t>0$ and $x \in V$. In what follows, $\partial_{t}$ denotes differentiation with respect to $t$, and $c_{q}$ and $c_{q}^{\prime}$ are positive constants which may depend on $q$ but are independent of $f$.

By definition of $\Gamma_{q}$ and since $\partial_{t}\left(u_{t}^{q}\right)=q u_{t}^{q-1}\left(\partial_{t} u_{t}\right)$, we have

$$
u_{t}^{q-2} \Gamma_{q}\left(u_{t}\right)=q u_{t}^{q-1}\left(H u_{t}\right)-H\left(u_{t}^{q}\right)=q u_{t}^{q-1}\left(\partial_{t}+H\right) u_{t}-\left(\partial_{t}+H\right)\left(u_{t}^{q}\right) ;
$$

since $\left(\partial_{t}+H\right) u_{t}=0$, it follows that

$$
\Gamma_{q}\left(u_{t}\right)=-u_{t}^{2-q}\left(\partial_{t}+H\right)\left(u_{t}^{q}\right)
$$


for $t>0$. Then

$$
\left(\mathcal{H}_{q}^{2} f\right)(x)=\int_{0}^{\infty} d t \Gamma_{q}\left(u_{t}\right)(x)=-\int_{0}^{\infty} d t u_{t}(x)^{2-q}\left(\partial_{t}+H\right)\left(u_{t}^{q}\right)(x) \leq f^{*}(x)^{2-q} J(x),
$$

where $f^{*}$ is as in Lemma 2.1 and we have set

$$
J(x):=-\int_{0}^{\infty} d t\left(\partial_{t}+H\right)\left(u_{t}^{q}\right)(x) \geq 0
$$

Therefore,

$$
\begin{aligned}
\sum_{x} m(x)\left(\mathcal{H}_{q}^{q} f\right)(x) & \leq \sum_{x} m(x) f^{*}(x)^{q(2-q) / 2} J(x)^{q / 2} \\
& \leq\left[\sum_{x} m(x) f^{*}(x)^{q}\right]^{(2-q) / 2}\left[\sum_{x} m(x) J(x)\right]^{q / 2}
\end{aligned}
$$

where the last step results from Hölder's inequality with exponents $r=$ $2 /(2-q)$ and $r^{\prime}=2 / q$. Lemma 2.1 yields

$$
\sum_{x} m(x) f^{*}(x)^{q} \leq c_{q}^{\prime}\|f\|_{q}^{q}
$$

Because $\sum_{x} m(x)(H g)(x)=0$ for any $g \in L^{1}$, we find that

$$
\begin{aligned}
\sum_{x} m(x) J(x) & =-\int_{0}^{\infty} d t\left(\sum_{x} m(x) \partial_{t}\left(u_{t}^{q}\right)(x)\right)=-\int_{0}^{\infty} d t \partial_{t}\left(\sum_{x} m(x)\left(u_{t}^{q}\right)(x)\right) \\
& \leq \sum_{x} m(x) f(x)^{q}=\|f\|_{q}^{q} .
\end{aligned}
$$

Substituting these results in (13) yields an estimate of type

$$
\sum_{x} m(x)\left(\mathcal{H}_{q}^{q} f\right)(x) \leq c_{q}\|f\|_{q}^{q},
$$

which proves the first statement of Theorem 1.3.

The proof of the second statement is a variation of the above argument which does not require Lemma 2.1. In fact, setting

$$
J_{t}(x):=-\left(\partial_{t}+H\right)\left(u_{t}^{q}\right)(x)
$$

and using (12), one gets

$$
\begin{aligned}
\left\|\Gamma_{q}^{1 / 2}\left(e^{-t H} f\right)\right\|_{q}^{q} & =\sum_{x} m(x) \Gamma_{q}^{q / 2}\left(u_{t}\right)(x) \\
& =\sum_{x} m(x) u_{t}(x)^{q(2-q) / 2} J_{t}(x)^{q / 2} \\
& \leq\left[\sum_{x} m(x) u_{t}(x)^{q}\right]^{(2-q) / 2}\left[\sum_{x} m(x) J_{t}(x)\right]^{q / 2}
\end{aligned}
$$


with the last step again by Hölder's inequality. For the expression in the first square brackets, we have $\sum_{x} m(x) u_{t}(x)^{q}=\left\|e^{-t H} f\right\|_{q}^{q} \leq\|f\|_{q}^{q}$. Next,

$$
\begin{aligned}
\sum_{x} m(x) J_{t}(x) & =-\sum_{x} m(x) \partial_{t}\left(u_{t}^{q}\right)(x)=-q \sum_{x} m(x) u_{t}^{q-1}(x) \partial_{t} u_{t}(x) \\
& \leq q\left\|u_{t}\right\|_{q}^{q / q^{\prime}}\left\|\partial_{t} u_{t}\right\|_{q}
\end{aligned}
$$

by Hölder's inequality with exponents $q^{\prime}$ and $q$, where $q^{-1}+\left(q^{\prime}\right)^{-1}=1$. Here $\left\|u_{t}\right\|_{q} \leq\|f\|_{q}$, while $\left\|\partial_{t} u_{t}\right\|_{q}=\left\|H e^{-t H} f\right\|_{q} \leq c_{q} t^{-1}\|f\|_{q}$ by analyticity of the semigroup $\left(e^{-s H}\right)_{s \geq 0}$ in $L^{q}$. Thus

$$
\sum_{x} m(x) J_{t}(x) \leq c_{q} t^{-1}\|f\|_{q}^{q} .
$$

Substitution of the estimates in (14) gives a bound of form $\left\|\Gamma_{q}^{1 / 2}\left(e^{-t H} f\right)\right\|_{q} \leq$ $c_{q} t^{-1 / 2}\|f\|_{q}$, and the proof of Theorem 1.3 is complete.

3. Proof of Theorem 1.1. Theorem 1.1 will follow from Theorem 1.3 via the following result. Define an averaging operator $A$ by setting

$$
(A f)(x)=\sum_{y \in B(x, 1)} f(y)
$$

for $x \in V$ and suitable functions $f: V \rightarrow \mathbb{R}$.

Proposition 3.1. Suppose that $(V, p, m)$ satisfies property (LD), and let $q \in(1,2]$. There exists a $c_{q}>0$ such that

$$
|\nabla f|^{2}(x) \leq c_{q}\left(A\left(\Gamma_{q} f\right)\right)(x)
$$

for all $x \in V$ and $0 \leq f \in L^{\infty}$. Moreover, there exists a $c_{q}^{\prime}>0$ such that

$$
\|A F\|_{q / 2} \leq c_{q}^{\prime}\|F\|_{q / 2}
$$

for any non-negative function $F$ on $V$.

Note that $q / 2 \leq 1$ in (15), and that we are using the notation $\|F\|_{r}:=$ $\left(\sum_{x} m(x)|F(x)|^{r}\right)^{1 / r}$ for $r \in(0,1]$.

Proposition 3.1 yields pointwise estimates

$$
\left|\nabla e^{-t H} f\right|^{2} \leq c A\left(\Gamma_{q}\left(e^{-t H} f\right)\right)
$$

and

$$
\begin{aligned}
(\mathcal{H} f)^{2} & =\int_{0}^{\infty} d t\left|\nabla e^{-t H} f\right|^{2} \\
& \leq c \int_{0}^{\infty} d t A\left(\Gamma_{q}\left(e^{-t H} f\right)\right) \\
& =c A\left(\int_{0}^{\infty} d t \Gamma_{q}\left(e^{-t H} f\right)\right)=c A\left(\left(\mathcal{H}_{q} f\right)^{2}\right)
\end{aligned}
$$


whenever $0 \leq f \in L^{1} \cap L^{\infty}$, so that Theorem 1.1 follows from Theorem 1.3 and from (15).

It remains to prove Proposition 3.1, and the rest of this section is devoted to this. We need two lemmas, in which we do not assume property (LD). The first lemma provides some explicit formulae for $\Gamma_{q}(f)$.

Lemma 3.2. For $q \in(1,2]$ and $0 \leq f \in L^{\infty}$ one has

$$
\begin{aligned}
& \Gamma_{q}(f)(x) \\
& \quad=\sum_{y} p(x, y)\left[q f(x)(f(x)-f(y))-f(x)^{2-q}\left(f(x)^{q}-f(y)^{q}\right)\right] \\
& \quad=q(q-1) \sum_{y: f(y) \neq f(x)} p(x, y)(f(x)-f(y))^{2} \int_{0}^{1} d u \frac{(1-u) f(x)^{2-q}}{((1-u) f(x)+u f(y))^{2-q}}
\end{aligned}
$$

for all $x \in V$.

Proof. Let $0 \leq f \in L^{\infty}$. The first expression for $\Gamma_{q}(f)(x)$ is a consequence of (10) and the equations

$$
\begin{gathered}
(H f)(x)=f(x)-\sum_{y} p(x, y) f(y)=\sum_{y} p(x, y)[f(x)-f(y)], \\
\left(H\left(f^{q}\right)\right)(x)=\sum_{y} p(x, y)\left[f(x)^{q}-f(y)^{q}\right] .
\end{gathered}
$$

To derive the second expression for $\Gamma_{q}(f)(x)$, note that a Taylor expansion of the function $t \mapsto t^{q}$ gives

$$
\begin{aligned}
t^{q}-s^{q} & =q s^{q-1}(t-s)+q(q-1) \int_{s}^{t} d \tau \tau^{q-2}(t-\tau) \\
& =q s^{q-1}(t-s)+q(q-1)(t-s)^{2} \int_{0}^{1} d u \frac{1-u}{((1-u) s+u t)^{2-q}}
\end{aligned}
$$

for any $s, t \geq 0$ with $s \neq t$, where the second step follows by a change of variable $\tau=(1-u) s+u t$. Remark that, in case $s=0$, the condition $q>1$ ensures that the integrals exist, and that we have excluded the case $s=t=0$, for which the second integral does not make sense.

If $f(y) \neq f(x)$, then setting $s=f(x), t=f(y)$ in (16) we find that

$$
\begin{aligned}
q f(x)(f(x)- & f(y))-f(x)^{2-q}\left(f(x)^{q}-f(y)^{q}\right) \\
& =f(x)^{2-q}\left[f(y)^{q}-f(x)^{q}-q f(x)^{q-1}(f(y)-f(x))\right] \\
& =f(x)^{2-q} q(q-1)(f(y)-f(x))^{2} \int_{0}^{1} d u \frac{1-u}{((1-u) f(x)+u f(y))^{2-q}} \\
& =q(q-1)(f(x)-f(y))^{2} \int_{0}^{1} d u \frac{(1-u) f(x)^{2-q}}{((1-u) f(x)+u f(y))^{2-q}} .
\end{aligned}
$$

Thus the second expression for $\Gamma_{q}(f)(x)$ follows from the first. 
REMARK. From the last expression of Lemma 3.2 and by observing that $(1-u)^{2-q} f(x)^{2-q} \leq((1-u) f(x)+u f(y))^{2-q}$, we see that

$$
\begin{aligned}
0 \leq \Gamma_{q}(f)(x) & \leq q(q-1) \sum_{y: f(y) \neq f(x)} p(x, y)(f(x)-f(y))^{2} \int_{0}^{1} d u(1-u)^{q-1} \\
& =(q-1)|\nabla f|^{2}(x),
\end{aligned}
$$

which establishes a sharper form of inequalities (11).

Lemma 3.3. Suppose that there exists $c \geq 1$ with

$$
m(y) \leq c m(x)
$$

for all $x, y \in V$ satisfying $x \sim y$. Then for each $q \in(1,2]$, there exists $c_{q}>0$, depending on $c$ and $q$, such that

$$
|\nabla f|^{2}(x) \leq c_{q} \sum_{y \in B(x, 1)}\left(\Gamma_{q} f\right)(y)
$$

for all $x \in V$ and $0 \leq f \in L^{\infty}$.

Proof. Let us write

$$
\begin{aligned}
|\nabla f|^{2}(x)= & \sum_{y: y \sim x, f(y)<f(x)} p(x, y)(f(x)-f(y))^{2} \\
& +\sum_{\substack{y: y \sim x, f(y)>f(x)\\
}} p(x, y)(f(x)-f(y))^{2} \\
= & E_{1}+E_{2} .
\end{aligned}
$$

To estimate $E_{1}$, observe that for $f(y)<f(x)$ one has $(1-u) f(x)+u f(y) \leq$ $f(x)$, hence

$$
\int_{0}^{1} d u \frac{(1-u) f(x)^{2-q}}{((1-u) f(x)+u f(y))^{2-q}} \geq \int_{0}^{1} d u(1-u)=1 / 2 .
$$

Therefore

$$
\begin{aligned}
E_{1} & \leq 2 \sum_{y: y \sim x} p(x, y)(f(x)-f(y))^{2} \int_{0}^{1} d u \frac{(1-u) f(x)^{2-q}}{((1-u) f(x)+u f(y))^{2-q}} \\
& =\frac{2}{q(q-1)} \Gamma_{q}(x)
\end{aligned}
$$

by Lemma 3.2. To estimate $E_{2}$, observe from (3) and the hypothesis of the lemma that

$$
p(x, y)=\frac{p(y, x) m(y)}{m(x)} \leq c p(y, x)
$$


whenever $y \sim x$. Using this and the inequality $(1-u) f(y)+u f(x) \leq f(y)$ for $f(y)>f(x)$, we obtain

$$
\begin{aligned}
E_{2} & \leq 2 c \sum_{y: y \sim x} p(y, x)(f(y)-f(x))^{2} \int_{0}^{1} d u \frac{(1-u) f(y)^{2-q}}{((1-u) f(y)+u f(x))^{2-q}} \\
& \leq \frac{2 c}{q(q-1)} \sum_{y: y \sim x}\left(\Gamma_{q} f\right)(y) .
\end{aligned}
$$

Since $B(x, 1)=\{x\} \cup\{y: y \sim x\}$, the lemma follows.

Proof of Proposition 3.1. The first statement of the proposition is immediate from Lemma 3.3. To see (15), observe that for a non-negative function $F$ and $r \in(0,1]$,

$$
\begin{aligned}
\|A F\|_{r}^{r} & =\sum_{x} m(x)\left(\sum_{y \in B(x, 1)} F(y)\right)^{r} \leq \sum_{x} m(x)\left(\sum_{y \in B(x, 1)} F(y)^{r}\right) \\
& =\sum_{y} F(y)^{r} \sum_{x \in B(y, 1)} m(x)=\sum_{y} F(y)^{r} m(B(y, 1)) \\
& \leq c_{0} \sum_{y} m(y) F(y)^{r}=c_{0}\|F\|_{r}^{r}
\end{aligned}
$$

where the last line used (6).

Remarks. Property (LD) holds if and only if

$$
\|A F\|_{r} \leq c\|F\|_{r}
$$

for some constants $r \in(0,1], c>0$, and all functions $F: V \rightarrow \mathbb{R}$. (The "if" statement is easily seen by taking $F$ to be the characteristic function of a single point.) We also remark that property (LD) implies boundedness of the operator $A$ in $L^{s}$ for all $s \in(1, \infty]$.

4. Proof of Corollary 1.2. Let $q \in(1,2]$. From (7) and the identity

$$
(I+t H)^{-1} f=\int_{0}^{\infty} d s e^{-s} e^{-s t H} f,
$$

it is easy to deduce an estimate of type

$$
\left\|\left|\nabla(I+t H)^{-1} f\right|\right\|_{q} \leq c t^{-1 / 2}\|f\|_{q}
$$

for all $f \in L^{q}$ and $t>0$. Replacing $f$ with $(I+t H) f$ yields

$$
\||\nabla f|\|_{q} \leq c t^{-1 / 2}\|(I+t H) f\|_{q} \leq c t^{-1 / 2}\|f\|_{q}+c t^{1 / 2}\|H f\|_{q}
$$

for all $t>0$; and (8) follows after optimizing with respect to $t$. 
Next, in case $-1 \notin \sigma_{L^{2}}(P)$, the spectral theorem for the self-adjoint contraction operator $P$ shows easily that

$$
\sup _{k \in \mathbb{N}} k\left\|H P^{k}\right\|_{2 \rightarrow 2}=\sup _{k \in \mathbb{N}} k\left\|(I-P) P^{k}\right\|_{2 \rightarrow 2}<\infty .
$$

An interpolation theorem of Blunck [3, Theorem 1.1] then shows that $\sup _{k \in \mathbb{N}} k\left\|H P^{k}\right\|_{q \rightarrow q}<\infty$ for any $q \in(1, \infty)$. Given $q \in(1,2]$, using this fact and (8) gives an estimate

$$
\left\|\left|\nabla P^{k} f\right|\right\|_{q} \leq c\left\|H P^{k} f\right\|_{q}^{1 / 2}\left\|P^{k} f\right\|_{q}^{1 / 2} \leq c^{\prime} k^{-1 / 2}\|f\|_{q}
$$

for all $k \in \mathbb{N}$. Corollary 1.2 is proved.

REMARKs. In general, for fixed $q \in(1, \infty)$, inequality (8) is equivalent to (7). To derive (7) from (8), replace $f$ by $e^{-t H} f$ in (8).

Moreover, in case $-1 \notin \sigma_{L^{2}}(P)$, for fixed $q$ all of the inequalities (7), (8) and (9) are equivalent. (Note that (9) implies (7) via the identity (5).)

5. Non-reversible case and random walks on groups. In this section we first generalize Theorem 1.3 to non-reversible random walks satisfying an $L^{2}$ sectorial estimate. We then study the particular case of non-reversible "centered" random walks on a discrete group, and obtain a Littlewood-Paley estimate in that case.

Let $p: V \times V \rightarrow[0,1]$ be an irreducible transition probability over a countable set $V$, and suppose that a function (measure) $m: V \rightarrow(0, \infty)$ is invariant for $p$ in the sense that

$$
\sum_{x} m(x) p(x, y)=m(y)
$$

for all $y \in V$. Assumption (17) is weaker than the reversibility condition (3). The Markov operator $P$ defined by (4) is a contraction in $L^{q}:=L^{q}(V ; m)$, $q \in[1, \infty]$, but is generally not self-adjoint in $L^{2}$. The adjoint operator $P^{*}$ is a Markov operator, however, corresponding to the transition probability $p^{*}(x, y):=m(y) p(y, x) / m(x)$. As before, we set $H:=I-P$, and define $\Gamma_{q}(f)$ by (10) for $q \in(1,2]$ and $0 \leq f \in L^{\infty}$. The inequalities $0 \leq \Gamma_{q}(f) \leq(q / 2) \Gamma_{2}(f)$ and the formulae of Lemma 3.2 remain valid. Theorem 1.3 generalizes as follows.

THEOREM 5.1. Let $(V, p, m)$ be as above, and suppose that for some $c>0$,

$$
|(H f, f)| \leq c \operatorname{Re}(H f, f)
$$

for all complex-valued functions $f \in L^{2}$. Then for each $q \in(1,2]$, the conclusions of Theorem 1.3 hold.

Proof. The sectorial estimate (18) implies that $\left(e^{-t H}\right)_{t \geq 0}$ is a bounded analytic semigroup in $L^{2}$, and that the operator $H$ has a bounded sectorial 
holomorphic functional calculus on $L^{2}$; for details see, for example, [1]. A standard interpolation argument shows that $\left(e^{-t H}\right)$ is a bounded analytic semigroup in $L^{q}$ for each $q \in(1, \infty)$.

For each $N \in \mathbb{N}$, define a "horizontal" Littlewood-Paley function $g_{N}(f)$ corresponding to a function $f$ by

$$
\begin{aligned}
g_{N}(f)(x) & :=\left[\int_{0}^{\infty} \frac{d t}{t}\left|t^{N}\left(\left(\partial_{t}\right)^{N} e^{-t H} f\right)(x)\right|^{2}\right]^{1 / 2} \\
& =\left[\int_{0}^{\infty} \frac{d t}{t}\left|\left((t H)^{N} e^{-t H} f\right)(x)\right|^{2}\right]^{1 / 2} .
\end{aligned}
$$

The "quadratic estimates" associated with the $L^{2}$ holomorphic functional calculus imply that $g_{N}$ is a bounded sublinear operator in $L^{2}$; see [1]. Then the arguments of [15, Chapter III], which use boundedness of $g_{N}$ in $L^{2}$, yield the conclusion of Lemma 2.1 for the maximal function $f^{*}(x):=$ $\sup _{t>0}\left|\left(e^{-t H} f\right)(x)\right|$. After these remarks, the proof of Theorem 5.1 is the same as the proof of Theorem 1.3.

We mention two closely related cases where Theorem 5.1 applies.

EXAmple 1 (Centeredness in Mathieu's sense). If $(V, p, m)$ is centered in the sense of Mathieu [14], then an estimate (18) holds, and hence Theorem 5.1 applies. See [14, Definition 2.1] for Mathieu's notion of centeredness, which involves a decomposition of $p$ into a sum of cycles.

In fact, if $(V, p, m)$ is centered, one proves in [14, Lemma 2.12] an estimate of type

$$
\left|\left(H f_{1}, f_{2}\right)\right|^{2} \leq c^{\prime}\left(H f_{1}, f_{1}\right)\left(H f_{2}, f_{2}\right)
$$

for all real-valued functions $f_{1}, f_{2} \in L^{2}$. But (19) is known to be equivalent to the sectorial estimate (18): see [13, Proposition I.2.17].

Under additional hypotheses on $(V, p, m)$, one can no doubt prove a comparison result analogous to Proposition 3.1, and then deduce a gradient Littlewood-Paley-Stein estimate analogous to Theorem 1.1. We shall not, however, pursue the details in this case.

EXAMPLE 2 (Homomorphism-centered random walks on a discrete group). Let $V$ be a finitely generated discrete group with identity element $e$, and $\widehat{p}: V \rightarrow[0,1]$ a probability on $V$, that is, $\sum_{x \in V} \widehat{p}(x)=1$. One can define a transition probability $p: V \times V \rightarrow[0,1]$ by

$$
p(x, y):=\widehat{p}\left(x y^{-1}\right), \quad x, y \in V,
$$

and the counting measure $m$, with $m(x):=1$ for all $x \in V$, is invariant 
for $p$. The associated Markov operator $P$ acts by convolution with $\widehat{p}$ :

$$
(P f)(x)=\sum_{y \in V} \widehat{p}\left(x y^{-1}\right) f(y)=(\widehat{p} * f)(x)
$$

for $x \in V$. Let $S:=\{x \in V: \widehat{p}(x)>0\}$ be the support of $\widehat{p}$. Let us assume that:

(i) $S$ is finite;

(ii) $\bigcup_{n=1}^{\infty} S^{n}=V$, where $S^{n}:=\left\{x_{1} \cdots x_{n}: x_{j} \in S\right\}$ denotes the set of all $n$-fold products of elements of $S$;

(iii) $\widehat{p}$ is homomorphism-centered, in the sense that

$$
\sum_{x} \widehat{p}(x) \eta(x)=0
$$

for every group homomorphism $\eta: V \rightarrow \mathbb{R}$.

It is easy to see that assumption (ii) is equivalent to irreducibility of $p$ on $V$.

Under assumptions (i)-(iii), the estimate (18) for $H=I-P$ is contained in [8] or [9]: see, for example, Propositions 3.2 and 3.3 of [8]. Hence Theorem 5.1 applies to $(V, p, m)$.

REMARKS.

- On finitely generated groups which have polynomial volume growth, Alexopoulos made a very detailed analysis of homomorphism-centered random walks in [2]. In comparison, results of $[9,8]$ for homomorphismcentered walks are less detailed, but apply to arbitrary finitely generated groups.

- For a probability on a finitely generated group, the relationship between homomorphism-centeredness and centeredness in Mathieu's sense is apparently not simple. Some results on this are developed in [14]. It seems from [14, Proposition 3.3], for example, that homomorphism-centeredness implies Mathieu-centeredness on some classes of groups (for example, nilpotent groups) but not on others (such as free groups).

The rest of this section is devoted to a Littlewood-Paley estimate in the situation of Example 2. For this situation it is convenient to use the following notion of gradient (cf. [16, Chapter VI]). Set $U:=S \cup S^{-1}$, so that $U$ is a finite set which generates the group $G$ (by assumption (ii)) and is symmetric $\left(U=U^{-1}\right)$. For $f: V \rightarrow \mathbb{R}$ put

$$
\nabla_{U} f(x):=\left(\sum_{u \in U}(f(u x)-f(x))^{2}\right)^{1 / 2}, \quad x \in V .
$$


Define a distance $d$ on $V$ by setting $d(x, x)=0$ and $d(x, y)=\inf \{n \in \mathbb{N}$ : $\left.x y^{-1} \in U^{n}\right\}$ for $x \neq y$, and let $B(x, r)=\{y \in V: d(x, y) \leq r\}$ for $r \geq 0$.

THEOREM 5.2. Let the probability $\widehat{p}$ satisfy assumptions (i)-(iii) on the finitely generated group $V$, and let $p(x, y):=\widehat{p}\left(x y^{-1}\right), P, H=I-P, U$ and $\nabla_{U}$ be as above. Then the operator $\mathcal{H}_{U}$ defined by

$$
\left(\mathcal{H}_{U} f\right)(x)=\left(\int_{0}^{\infty} d t\left|\nabla_{U} e^{-t H} f\right|^{2}(x)\right)^{1 / 2}, \quad x \in V,
$$

is bounded in $L^{q}, q \in(1,2]$. For $q \in(1,2]$ one also has an estimate

$$
\left\|\nabla_{U} e^{-t H} f\right\|_{q} \leq c_{q} t^{-1 / 2}\|f\|_{q}
$$

for all $t>0$ and $f \in L^{q}$. If , in addition, $\sigma_{L^{2}}(P) \subseteq\{z \in \mathbb{C}:|z|<1\} \cup\{1\}$, then for $q \in(1,2]$ one has an estimate

$$
\left\|\nabla_{U} P^{k} f\right\|_{q} \leq c_{q}^{\prime} k^{-1 / 2}\|f\|_{q}
$$

for all $k \in \mathbb{N}$ and $f \in L^{q}$.

To prove Theorem 5.2 we need the following analogue of Proposition 3.1.

Lemma 5.3. Let $q \in(1,2]$. There exist constants $c_{q}>0$ and $N \in \mathbb{N}$ such that

$$
\left|\nabla_{U} f\right|^{2}(x) \leq c_{q} \sum_{y \in B(x, N)} \Gamma_{q}(f)(y)
$$

for all $x \in V$ and $f: V \rightarrow[0, \infty)$.

Proof. By assumptions (i) and (ii), we can choose an $N \in \mathbb{N}$ such that

$$
U=S \cup S^{-1} \subseteq \bigcup_{j=1}^{N} S^{j} .
$$

Put $\beta:=\inf \{\widehat{p}(x): x \in S\}>0$. Take $f: V \rightarrow[0, \infty), x \in V, u \in U$, and set $y=u x$. To estimate $(f(y)-f(x))^{2}$ we consider two cases.

CASE 1: $f(y)<f(x)$. By definition of $N$, there exist $k \in\{1, \ldots, N\}$ and $v_{1}, \ldots, v_{k} \in S$ such that $u^{-1}=v_{1} \cdots v_{k}$. Set $x_{0}:=x$ and $x_{j}:=v_{j}^{-1} \cdots v_{1}^{-1} x$. The sequence $x_{0}, x_{1}, \ldots, x_{k}$ satisfies $x_{0}=x, x_{k}=y$ and

$$
p\left(x_{j}, x_{j+1}\right)=\widehat{p}\left(v_{j+1}\right) \geq \beta
$$

for $j \in\{0,1, \ldots, k-1\}$. Let $J$ be the set of those $j \in\{0,1, \ldots, k-1\}$ for which $f\left(x_{j+1}\right) \leq f\left(x_{j}\right)$, and observe that

$$
|f(y)-f(x)|=f(x)-f(y) \leq \sum_{j \in J}\left(f\left(x_{j}\right)-f\left(x_{j+1}\right)\right) .
$$


For $j \in J$, since $(1-u) f\left(x_{j}\right)+u f\left(x_{j+1}\right) \leq f\left(x_{j}\right)$ for $u \in(0,1)$, we see from the last expression of Lemma 3.2 that

$$
\begin{aligned}
\left(f\left(x_{j}\right)-f\left(x_{j+1}\right)\right)^{2} & \leq 2\left(f\left(x_{j}\right)-f\left(x_{j+1}\right)\right)^{2} \int_{0}^{1} d u \frac{(1-u) f\left(x_{j}\right)^{2-q}}{\left((1-u) f\left(x_{j}\right)+u f\left(x_{j+1}\right)\right)^{2-q}} \\
& \leq \frac{2 \beta^{-1}}{q(q-1)} \Gamma_{q}(f)\left(x_{j}\right) .
\end{aligned}
$$

These estimates imply that there is a constant $c>0$, depending only on $q$, $\beta$ and $N$, such that

$$
(f(y)-f(x))^{2} \leq c \sup _{j \in J} \Gamma_{q}(f)\left(x_{j}\right) .
$$

Now $d\left(x_{j}, x\right) \leq N-1$ for all $j \in J$, so that

$$
(f(y)-f(x))^{2} \leq c \sum_{z \in B(x, N-1)} \Gamma_{q}(f)(z) .
$$

CASE 2: $f(y)>f(x)$. Since $x=u^{-1} y$ with $u^{-1} \in U$, we may apply Case 1 with the roles of $x$ and $y$ reversed, to obtain

$$
(f(y)-f(x))^{2} \leq c \sum_{z \in B(y, N-1)} \Gamma_{q}(f)(z) \leq c \sum_{z \in B(x, N)} \Gamma_{q}(f)(z) .
$$

Lemma 5.3 follows from Cases 1 and 2 .

Proof of Theorem 5.2. The first two statements of the theorem are straightforward deductions from Theorem 5.1 and Lemma 5.3; we leave details to the reader (cf. the proof of Theorem 1.1).

To get (21), we use the fact (see for example [8, Corollary 1.5]) that under the additional assumption on $\sigma_{L^{2}(P)}$ one has

$$
\sup _{k \in \mathbb{N}} k\left\|H P^{k}\right\|_{q \rightarrow q}<\infty
$$

for each $q \in(1, \infty)$. Then $(21)$ follows by the same argument as Corollary 1.2 .

Acknowledgements. This work was financially supported by the ARC (Australian Research Council). I thank X. T. Duong for a number of valuable discussions.

\section{References}

[1] D. Albrecht, X. T. Duong and A. McIntosh, Operator theory and harmonic analysis, in: Instructional Workshop on Analysis and Geometry, Part III (Canberra, 1995), Proc. Centre Math. Appl. Austral. Nat. Univ. 34, Austral. Nat. Univ., Canberra, 1996. 
[2] G. Alexopoulos, Random walks on discrete groups of polynomial volume growth, Ann. Probab. 30 (2002), 723-801.

[3] S. Blunck, Analyticity and discrete maximal regularity on $L_{p}$-spaces, J. Funct. Anal. 183 (2001), 211-230.

[4] T. Coulhon and X. T. Duong, Riesz transforms for $1 \leq p \leq 2$, Trans. Amer. Math. Soc. 351 (1999), 1151-1169.

[5] - - - Riesz transform and related inequalities on noncompact Riemannian manifolds, Comm. Pure Appl. Math. 56 (2003), 1728-1751.

[6] T. Coulhon, X. T. Duong and X. D. Li, Littlewood-Paley-Stein functions on complete Riemannian manifolds for $1 \leq p \leq 2$, Studia Math. 154 (2003), 37-57.

[7] T. Coulhon, A. Grigor'yan and F. Zucca, The discrete integral maximum principle and its applications, Tohoku Math. J. 57 (2005), 559-587.

[8] N. Dungey, Time regularity for random walks on locally compact groups, Probab. Theory Related Fields 137 (2007), 429-442.

[9] - Properties of random walks on discrete groups: time regularity and off-diagonal estimates, Bull. Sci. Math., to appear.

[10] - - A note on time regularity for discrete time heat kernels, Semigroup Forum 72 (2006), 404-410.

[11] Y. Han and E. Sawyer, Littlewood-Paley theory on spaces of homogeneous type and the classical function spaces, Mem. Amer. Math. Soc. 110 (1994), no. 530.

[12] N. Lohoué, Estimations des fonctions de Littlewood-Paley-Stein sur les variétés riemanniennes à courbure non positive, Ann. Sci. École Norm. Sup. 20 (1987), 505544 .

[13] Z. Ma and M. Röckner, Dirichlet Forms, Springer, Berlin, 1992.

[14] P. Mathieu, Carne-Varopoulos bounds for centered random walks, Ann. Probab. 34 (2006), 987-1011.

[15] E. M. Stein, Topics in Harmonic Analysis Related to the Littlewood-Paley Theory, Ann. of Math. Stud. 63, Princeton Univ. Press, Princeton, 1970.

[16] N. T. Varopoulos, L. Saloff-Coste and T. Coulhon, Analysis and Geometry on Groups, Cambridge Tracts in Math. 100, Cambridge Univ. Press, Cambridge, 1992.

[17] W. Woess, Random Walks on Infinite Graphs and Groups, Cambridge Tracts in Math. 138, Cambridge Univ. Press, Cambridge, 2000.

Department of Mathematics

Macquarie University

Sydney, NSW 2109, Australia

E-mail: ndungey@ics.mq.edu.au 